\title{
Prediction of mechanical properties of porous CFRP specimens by ANNs and X-ray CT data
}

\author{
Antonios Stamopoulos ${ }^{1},{\text { Konstantinos } \text { Tserpes }^{1 *} \text {, and Argyris Dentsoras }}^{2}$ \\ ${ }^{1}$ Laboratory of Technology \& Strength of Materials, Department of Mechanical Engineering \& \\ Aeronautics, University of Patras, Patras 26500, Greece \\ ${ }^{2}$ Machine Design Laboratory, Department of Mechanical Engineering \& Aeronautics, University of \\ Patras, Patras 26500, Greece
}

\begin{abstract}
Carbon fiber reinforced plastics (CFRPs) have evolved into the primary material for several lightweight structures. However, despite their extensive use and the quality amelioration, CFRPs remain susceptible to a variety of manufacturing defects, the most common of which are the pores. Predictive tools capable of correlating the mechanical properties of CFRP parts with the characteristics of defects as derived from non-destructive testing (NDT) techniques or even further with the manufacturing parameters could serve as an effective tool for the quality control of CFRP structural parts. In the present paper, the characteristics of pores as evaluated by X-ray Computed Tomography (CT) have been correlated with the matrixdominated mechanical properties of unidirectional porous CFRP specimens using Artificial Neural Networks (ANN). Thirty (30) porosity scenarios have been created and given as input to the numerical model. That multi-scale numerical model, which had been validated experimentally, has been used for training the ANN model. The predictions of the ANN agree very well with results from mechanical tests. Moving one step forward, a second ANN has been developed to correlate the autoclave pressure directly with the mechanical properties of the CFRP specimens. The validity of the latter ANN depends on the accuracy of the relation between the autoclave pressure and the characteristics of the pores. The present work represents a step towards the development of effective quality control tools for composite materials.
\end{abstract}

\section{Introduction}

In the recent years, significant progress is observed in the field of manufacturing of CFRP materials aiming on producing high quality parts for use in the aeronautic industry. Nevertheless, CFRPs remain susceptible to of a range of factors (e.g. temperature, autoclave pressure) that influence severely the quality of these materials. Consequently, a wide range of defects are introduced during this phase, namely porosity, fiber misalignment or delaminations. From these defects, porosity is considered unavoidable and affects severely all the matrix-dominated properties of composite materials [1-2]. To this end, knowing the porosity characteristics and their effect to the mechanical properties of the materials during the design phase is crucial as it is the subject of a number of previous works on this field [3-4]. All the previous research was focused either on the investigation 
of the influence of porosity on the mechanical behaviour of composite materials by mechanical testing or on the development of FE models to describe and predict the failure mechanisms.

Considering the time consuming and price inefficiency of the previously mentioned methodologies, in the present paper, presented are two artificial neural networks (ANNs) which were trained using an FE methodology which exploits optimally the output of X-Ray CT scan and image analysis. The accuracy of the two ANNs is validated with mechanical tests. In the following sections the workflow, the experimental procedures, the development of the FE models using the methodology developed by Tserpes et al. [5], the training and the performance of the ANNs are described.

\section{Workflow}

The workflow of the present work is presented in the following flow-chart of Table 1. As seen there, the first phase of the present work includes the manufacturing of porous CFRP laminates, the X-Ray computed tomography application for detecting the porosity developed inside the laminates and the quantification of it as seen in [6]. Consequently, for training the ANNs, the creation of porosity scenarios follows which is also the input to the previously mentioned numerical methodology [5]. The predictions of this FE models in the form of mechanical properties are used for training the ANNs.

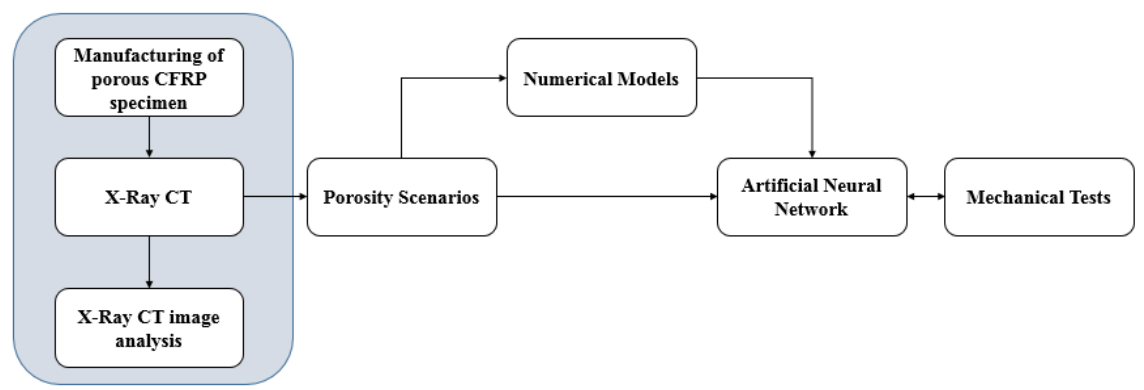

Fig. 1 : Flow chart of the present work

This way, throughout this procedure, a correlation between the porosity characteristics described in each scenario and the mechanical properties of the porous laminates is generated. Moreover, another relation between the variation of the autoclave pressure and the mechanical properties can also be established.

\section{Experimental}

\subsection{Materials and Manufacturing}

The CFRP specimens were made of prepreg UD CFRP plates using the EHkF 420 epoxy resin and the HTA $24 \mathrm{k}$ carbon fibers. The weight fraction of the resin was $35 \%$. Aiming to introduce different porosity levels and characteristics to the material during the manufacturing process, 4 different autoclave pressures were applied, namely $0.1 \mathrm{MPa}, 0.35$ $\mathrm{MPa}, 0.6 \mathrm{MPa}$ and $1 \mathrm{MPa}$. These pressures correspond to the highest, lowest and most usual production cases, thus they were labelled as Reference material, Minimum, Medium and Extensive porosity plates. Moreover, during the manufacturing process, the temperature cycle and the dwell were the same in every case. More information about the curing cycles and the materials can be found in [5-7]. 


\subsection{Porosity Quantification}

\subsubsection{X-Ray CT tests}

In order to detect and quantify the porosity developed inside the CFRP plates manufactured, X-Ray Computed Tomography was applied in samples cut from each plate respectively. The sample dimensions were roughly $15 \mathrm{~mm}$ long, $10 \mathrm{~mm}$ wide and $2.75 \mathrm{~mm}$ thick. A fundamental assumption made in this point was that these samples are representative of the whole CFRP plates they were cut from. More information about the scanning apparatus and the tests can also be found in [6].

\subsubsection{CT image analysis}

The output of the X-Ray CT tests in the form of a 3D image was analysed using the VG Studio MAX software [8]. An initial calibration of the analysis parameters was conducted along with a validation using optical microscopy as seen in [5]. The porosity developed inside the samples was detected and fully characterized. The difference between the porosity samples in terms of pores' size and population can be seen in Fig.2

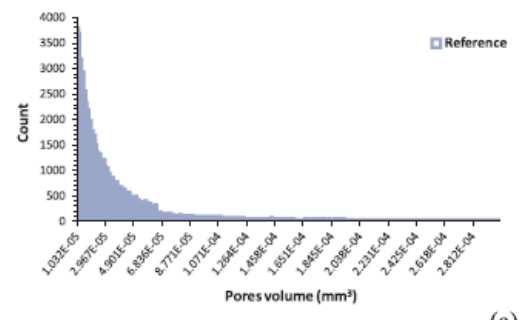

(a)

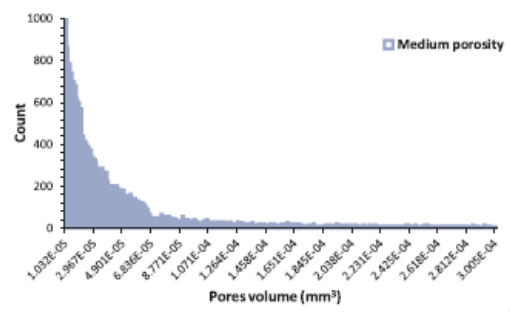

(c)

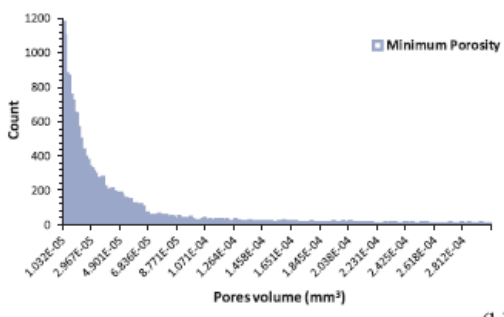

(b)

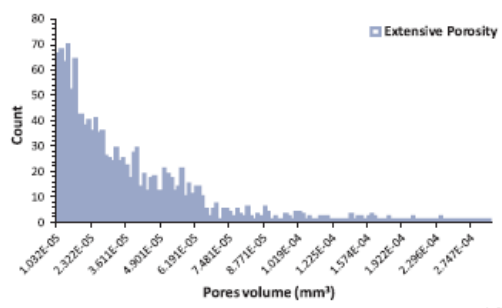

(d)

Fig. 2 : The pore population and size in the Reference material (a), Minimum (b), Medium (c) and Extensive porosity (d) samples.

The overall pore content of each sample in terms of volume fraction was $0.82,1.56 \%$, $1.62 \%$ and $4.22 \%$ respectively. Moreover, the number of larger pores tends to increase while the population of smaller pores tends to decrease as the autoclave pressure is reduced.

\subsection{Mechanical Testing}

The mechanical tests of the composite materials were conducted mainly for two reasons; to investigate the influence of different porosity levels to the matrix dominated properties and to compare them with the predictions of the ANN developed. To this end, transverse tension, three point bending and short beam strength (ILSS) tests were conducted using the ASTM D3039 [9], ISO 15124 [10] and ASTM D2344 [11] standards. 
Consequently, the obtained mechanical properties were the transverse strength $\left(F_{T T}\right)$, transverse stiffness $\left(E_{T}\right)$, flexural strength $\left(F_{f}\right)$, flexural modulus $\left(E_{f}\right)$ and short beam strength $\left(F_{S B S}\right)$. More information about the specimen of each test, procedure and findings can be also found in [6].

\section{Multi-scale Numerical Methodology}

\subsection{Porosity Scenarios}

In order to produce enough training samples to train the ANNs, the numerical methodology described in [5] was utilized. This methodology exploits the outcome of the image analysis of a CT scan to create FE models of small and larger pores dispersed arbitrarily inside the matrix of CFRPs. Due to the lack of experimental data concerning the porosity characteristics caused by the variance of the autoclave pressure in the range between 0.1 (Extensive porosity) and $1 \mathrm{MPa}$ (Reference material), the porosity characteristics (small and large pores volume fraction, average gap between neighbouring pores and number of pores) were related to the autoclave pressure using regression lines. An example of the regression can as seen in Fig.3. where the small and large pores volume fraction related to the autoclave pressure is presented.

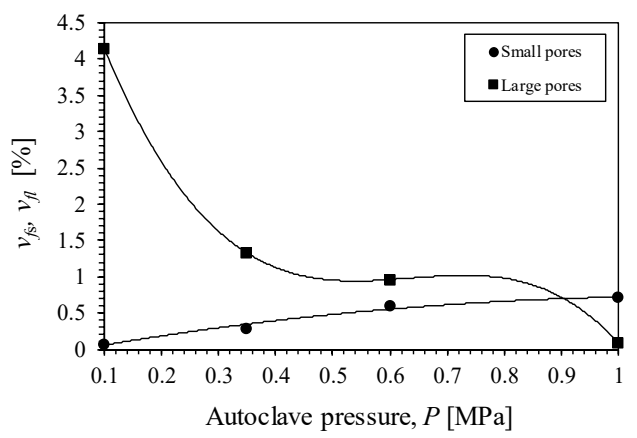

Fig. 3 : Polynomial regression of the small and large pores volume fraction for producing scenarios as input to the numerical methodology.

Table 1 : Functions between pores' characteristics and the autoclave pressure

\begin{tabular}{|c|c|}
\hline Pores Characteristic & $\begin{array}{l}\text { Function between the pores characteristic and the } \\
\text { autoclave pressure }(P)\end{array}$ \\
\hline Small pores volume fraction $(v f \%)$ & $v_{f s}(P)=-0.692 P^{2}+1.4555 P-0.0776$ \\
\hline Volume fraction of large pores, $v_{f R}[(2)$ & $v_{f l}(P)=-22.877 P^{4}+43,541 P^{2}-27,001 P+6.4276$ \\
\hline $\begin{array}{l}\text { Number of pores with size } 0-0.2 \mathrm{~mm}, \\
N_{1}\end{array}$ & $N_{1}(P)=65958 F^{5}-102511 P^{2}+175706 F+2160$ \\
\hline $\begin{array}{l}\text { Number of pores with size } 0.2-0.6 \mathrm{~mm} \text {, } \\
\qquad N_{2}\end{array}$ & $M_{2}(P)=-4711,4 P^{2}+7622,2 P+900.57$ \\
\hline $\begin{array}{l}\text { Number of pores with size } 0.6-0.99 \\
\mathrm{~mm}, N_{3}\end{array}$ & $N_{g}(F)=-515,9 F^{2}+163,99 F^{2}+967,25 F+266,95$ \\
\hline Average gap $G[\mathrm{~mm}]$ & $G(P)=-0.112 P^{2}+0.1624 P^{2}-0.0909 P+0.1541$ \\
\hline
\end{tabular}

All the 30 scenarios were selected by dividing the range of the available autoclave pressure in 30 equally spaced points while the pressures in which the Minimum and Medium porosity plates were manufactured were excluded from the selection and used as new cases 
for validating the ANNs' predictions. The polynomial equations are presented in the following table:

\subsection{Multi-scale Numerical Methodology}

The previously mentioned multi-scale numerical methodology was developed and described in [5]. The principals of this methodology are explained in Fig.4 by means of flow chart.

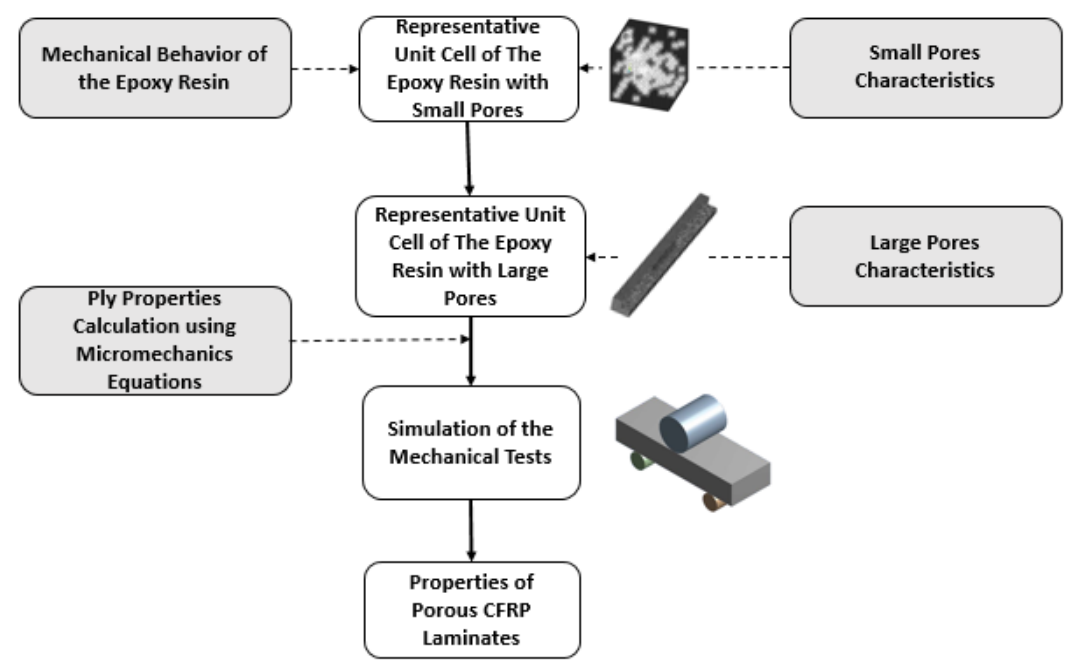

Fig. 4: Flow chart of the developed numerical methodology.

Each simulation level (RUC of small pores, RUC of large pores) provides the next level with input for calculating both the mechanical behaviour and the properties either of the porous epoxy resin (RUCs) or the lamina (Simulation of mechanical tests). To this end, 30 geometries of each of the first two levels were created and subjected to tension, compression and planar shear. This procedure derived 90 simulations in the first, 90 in the second and 90 at the third simulation level (three point bending, short beam shear and transverse tension), making a total of 270 simulations. All these simulations were performed in ANSYS APDL [12]. For accomplishing this goal, approximately 7 months were required using a X5460@3.17 GHz dual processor computer with 64 GB of RAM. The results of this procedure were the mechanical properties of the porous laminates discussed also in section 3.2 of the present paper.

\section{Artificial Neural Networks}

An artificial neural network (ANN) is mostly an arithmetical system which imitates the cerebral activity of biological systems, thus it learns by the examples given. It is capable of giving predictions for a new case of input within the range of the examples with which it was trained in a very short period of time. Thus they are widely used in a variety of problems that need to be simplified, including composite materials' technological problems [13-14]. In the present work, 2 ANNs were developed using MatLab NN Tool [15] as seen in Fig. 5. The first one (ANN1) connects the pores' characteristics with the mechanical properties (aiming to substitute the time consuming FE models) and the second one 
(ANN2) connects the autoclave pressure with the mechanical properties respectively (avoiding the X-Ray CT scan and image analysis).

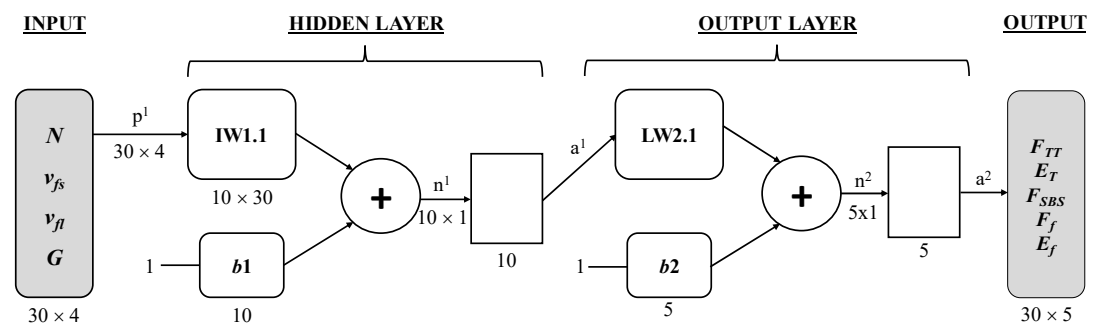

(a)

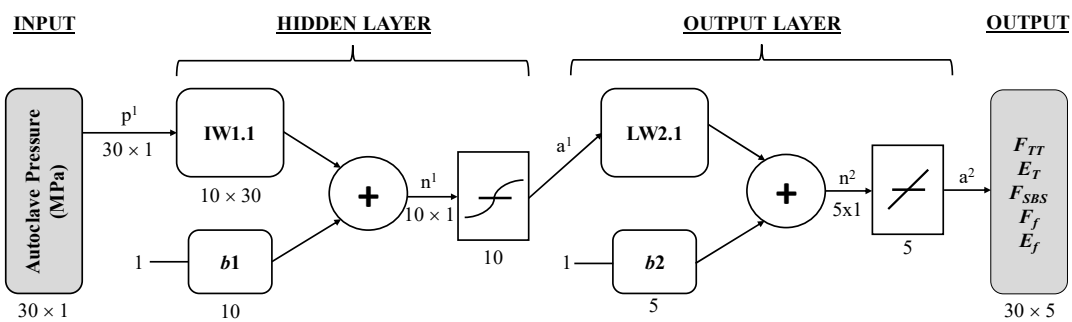

Fig. 5 : The ANN1 (a) and ANN2 (b) morphology

The ANNs are static, thus the sequence precedence of the input values has not been considered. The feed-forward-back-propagation training method was utilized along with the Levenberg-Marquand training algorithm. From the examples that were available, $70 \%$ was used for training, $15 \%$ for validation and $15 \%$ for training. The final architecture of the layers and the transfer functions (tansig at the first and purelin at the second layer) were defined after a trial and error procedure for reducing the mean square error and the number of epochs required for the training of the ANNs. Due to the relatively low number of available training examples, the two ANN architectures were trained several times using each time different examples for both training, validation and testing and the obtained results were averaged. These values are considered, in the present work, as the actual predictions of the ANNs.

\section{Comparison with Mechanical Tests}

As the Minimum and Medium porosity characteristics were given as a new input to the developed ANNs, the corresponding mechanical tests of the specimens of these two porosity levels were used to compare the predictions of the ANNs with the actual case. To this end, in Fig.6 the comparison between ANN1, ANN2 and the mechanical tests is presented. In (a) and (b), the transverse tensile properties (strength and stiffness) are presented, in (c) and (d) the flexural (strength and modulus) while in (e ) the short beam strength. It must also be pointed the fact for providing output from a new input, each ANN requires less than 1 minute. 


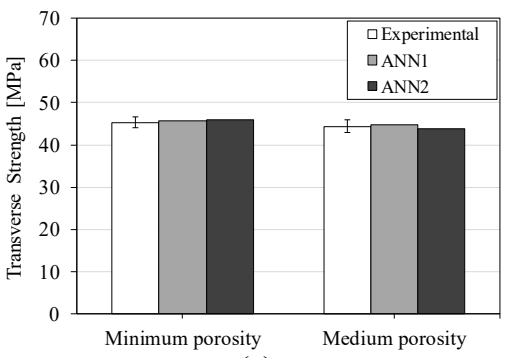

(a)

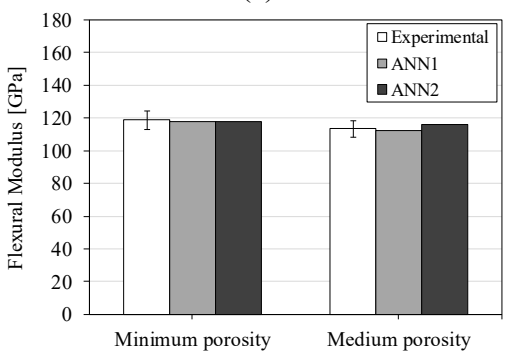

(c)

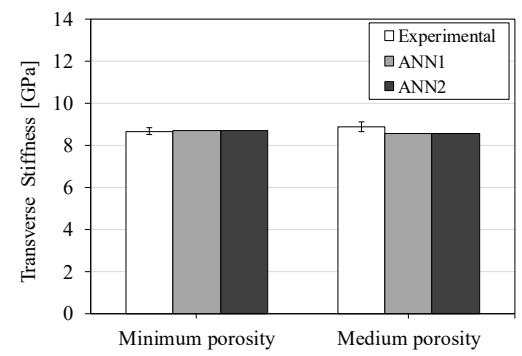

(b)

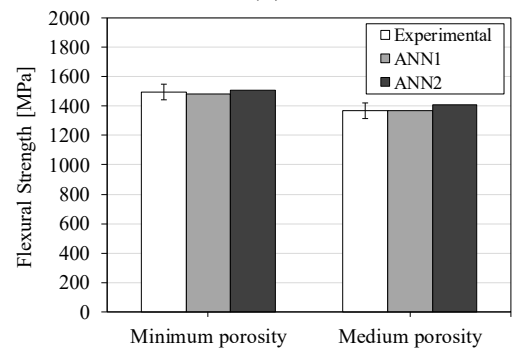

(d)

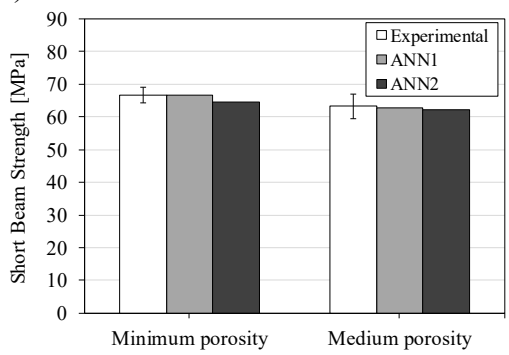

(e)

Fig. 6 : Comparison of the mechanical properties derived by mechanical testing and the predictions of the ANNs.

As seen in Fig.6, a good agreement between the ANNs was achieved as they both derive predictions for both the Minimum and Medium porosity levels. However, the predictions of the ANN which correlates the autoclave pressure with the mechanical properties derives less accurate predictions compared to the one that correlates the porosity characteristics with the corresponding mechanical properties, especially in the cases of the flexural and the interlaminar shear test.

\section{Conclusions}

In the present work two artificial neural networks were developed for predicting the matrix-dominated mechanical properties of porous CFRP laminates, having as input either porosity characteristics or the autoclave pressure value. By the comparison with mechanical tests, the good agreement achieved can easily be concluded. Nevertheless, the ANN2 derives less accurate results compared to ANN1 due to the fact that the porosity characteristics were related to the autoclave pressure throughout polynomial regression while in fact this relation is not certain and derives some error. Generally though, the predictions were within the limits of the standard deviation of the mechanical tests. The time required by the ANNs to predict the properties of a new case compared to the time needed from the FE models of the numerical methodology described previously, leads to the conclusion that the ANNs may be a first step towards a fast and effective quality 
assessment of CFRP parts. However, in any case, the input must be ascertained, thus the analysis of the X-Ray CT images must be accurate in order to derive robust information that can be used as input both in training and validation of the ANNs.

\section{References}

1. S. Sisodia, E.K. Gamstedt, F. Edgren, J. Varna. J. Compos. Mater. 49, 17 (2014)

2. H. Koushyar, Effects of variation in autoclave pressure, cure temperature and vaccum application time on the porosity and mechanical properties of a carbon/epoxy composite, Master thesis (Department of Mechancial Engineering, Wichita University, USA, 2009)

3. B. Drach, I. Tsukrov, T. Gross, Dietrich, K. Weidenmann, R. Piat, T. Bohlke. J. Sol. Struct. 48 (2011)

4. I. Balać, K. Čolić, M. Milovančević, P. Uskoković, M. Zrilić. FME Trans. 40 (2012)

5. K.I. Tserpes, A.G. Stamopoulos, Sp. Pantelakis. Comp. Part B. 102 (2016)

6. A.G. Stamopoulos, K.I. Tserpes, D. Vavrik, P. Prucha. J. Compos. Mater. 50, 15 (2015)

7. A.G. Stamopoulos, K.I. Tserpes, Sp. Pantelakis. Theor. And Ap. Fract. Mech. https://doi.org/10.1016/j.tafmec.2017.04.020 (2017)

8. VG Studio MAX Reference Manual v2.2, Volume Graphics GmbH, 1998-2014.

9. Standard test method for tensile properties of polymer matrix composite materials. Designation: D3039/D3039M-95a. American society for testing of materials (ASTM)

10. ISO 14125:1998, Fibre-reinforced Plastic Composites, Determination of Flexural Properties, English Version of DIN EN ISO 14125

11. Standard test method for short-beam strength of polymer matrix composite materials and their laminates. Designation: D2344/ D2344M-00, American society for testing of materials (ASTM)

12. ANSYS Mechanical Users Guide, Release 15 (2013)

13. K.M. Saridakis, A.C. Chasalevris, C.A. Papadopoulos. Comput Struct 86, 38 (2008)

14. G. Balokas, S. Czichon, R. Rolfes. Compos. Struct. 183, 1 (2018)

15. H. Demuth, M. Beale. Neural network toolbox for the use with Matlab. User's guide, version 4. The MathWorks (2002) 